\section{Insatisfação com a imagem corporal em mulheres obesas: a importância do transtorno da compulsão alimentar periódica}

Body image dissatisfaction in obese women: the importance of binge eating disorder

Rodrigo O. Moreira ${ }^{1}$, Ana Patrícia C. Batista ${ }^{2}$

1 Médico. Pesquisador, Grupo de Obesidade e Transtornos Alimentares (GOTA), Instituto Estadual de Diabetes e Endocrinologia (IEDE)/Instituto de Psiquiatria Universidade Federal do Rio de Janeiro (IPUB/UFRJ), Rio de Janeiro, RJ. Pesquisador, Escola Médica de PósGraduação, Pontifícia Universidade Católica do Rio de Janeiro (PUCRJ), Rio de Janeiro, RJ. ${ }^{2}$ Pós-graduanda em Endocrinologia, Escola Médica de Pós-Graduação, PUCRJ, Rio de Janeiro, RJ.

\section{Prezados Editores,}

Gostaríamos de apresentar alguns dados que chamaram nossa atenção em um dos trabalhos que vêm sendo desenvolvidos por nosso grupo. O transtorno da compulsão alimentar periódico (TCAP) é o transtorno alimentar mais prevalente na obesidade. Ele é caracterizado por episódios recorrentes de "ataques de comer”, associados à falta de controle e sem a presença de mecanismos compensatórios. Pacientes com TCAP geralmente são caracterizados por um perfil psicopatológico mais grave do que obesos comuns, com uma maior prevalência de diversas síndromes psiquiátricas, incluindo transtornos depressivos e ansiosos ${ }^{1}$.

Sabe-se, atualmente, que pacientes com transtornos alimentares, principalmente a anorexia e a bulimia nervosa, caracterizam-se por grandes alterações na percepção da imagem corporal. Nos pacientes obesos com TCAP, existe ainda uma discussão sobre se a insatisfação com a imagem corporal seria decorrente primariamente do TCAP, isto é, da perturbação alimentar, ou se seria secundária ao excesso de peso apresentado pelos pacientes. Alguns autores sugerem que as alterações corporais relacionadas com a obesidade poderiam levar às alterações psicopatológicas capazes de desencadear quadros graves de distúrbios da imagem corporal e transtornos psiquiátricos típicos ${ }^{2}$.

Avaliamos consecutivamente 156 mulheres obesas (índice de massa corporal - IMC $=39,8 \pm 6,4)$, com idade entre 18 e 77 anos (39,1 $\pm 11,9)$, que procuraram tratamento para emagrecer no Grupo de Obesidade e Transtornos Alimentares do Instituto Estadual de Diabetes e Endocrinologia do Rio de Janeiro. O protocolo foi aprovado pelo comitê de ética em pesquisa da instituição. O diagnóstico do TCAP foi realizado por um psiquiatra treinado, de acordo com a versão brasileira do Structured Clinical Interview for DSM$I V^{3}$. A insatisfação com a imagem corporal foi avaliada utilizando-se a versão brasileira do Body Shape Questionnaire (BSQ).

Foram identificadas 46 pacientes com TCAP (29,4\%). Mulheres com TCAP apresentavam, em relação a mulheres sem TCAP, mesma idade (38,4 \pm $8,4$ versus $39,3 \pm 12,0$, respectivamente; $p=0,67)$ e mesmo IMC $(39,7 \pm 5,9$ versus $39,8 \pm 6,8$, respectivamente; $\mathrm{p}=0,96)$. Em relação à imagem corporal, mulheres obesas com TCAP apresentavam níveis significativamente mais elevados no BSQ do que mulheres sem TCAP $(148,9 \pm 26,7$ versus $133,8 \pm 29$, 8 , respectivamente; $\mathrm{p}=0,003)$.

Nossos resultados levam a algumas considerações interessantes. Parece que a insatisfação corporal em mulheres obesas está relacionada muito mais com a presença de TCAP do que com o próprio excesso de peso. Esses valores mais elevados do BSQ podem ser considerados como um reflexo de fatores psicopatológicos relacionados ao TCAP, tais como: baixa auto-estima, depressão, preocupação extrema com a aparência, e não com o peso corporal ${ }^{4}$. Os achados demonstrados por Sarwer et al. ${ }^{5}$ e Foster et al. ${ }^{6}$ foram semelhantes aos nossos estudos, sugerindo que o IMC não apresenta relação com a insatisfação corporal.

A importância dos nossos achados reside no fato de que muitos dos artigos que avaliam imagem corporal levam em conta diversas variáveis sociodemográficas e antropométricas. É importante ressaltar que um transtorno como o TCAP pode estar presente em até $30 \%$ de uma população e que pode interferir, de maneira determinante, na avaliação da imagem corporal de pacientes obesas, devendo ser sempre levado em conta como fator de confusão.

\section{Referências}

1. Fontenelle LF, Vltor Mendlowicz M, de Menezes GB, Papelbaum M, Freitas SR, Godoy-Matos A, et al. Psychiatric comorbidity in a Brazilian sample of patients with binge-eating disorder. Psychiatry Res. 2003;119(1-2):189-94.

2. Kessler RC, McGonagle KA, Zhao S, Nelson CB, Hughes M, Eshleman S, et al. Lifetime and 12-month prevalence of DSM-III_R psychiatric disorders in the United States. Results from the National Comorbidity Survey. Arch Gen Psychiatry. 1994;51(1):8-19.

3. First MB, Spitzer RL, Gibbom M, Willimas JBW. Structured clinical interview for DSM-IV axis I disorders: patients edition (SCID-IV, Version 2.0). New York: Biometrics Research Department, New York State Psychiatric Institute; 1996.

4. Cash TF, Fleming EC. Body image and social relations. In: Cash TF, Pruzinsky T, eds. Body images: a handbook of theory, research, and clinical practice. New York: Guilford: 2002. p. 277-86. 
5. Sarwer DB, Wadden TA, Foster GD. Assessment of body image dissatisfaction in obese women: specificity, severity, and clinical significance. J Consult Clin Psychol. 1998;66(4):651-4.

6. Foster GD, Wadden TA, Vogt RA. Body image in obese women before, during, and after weight loss treatment. Health Psychol. 1997;16(3):226-9.

\section{Correspondência:}

Rodrigo O. Moreira, Instituto Estadual de Diabetes e Endocrinologia,

Rua Moncorvo Filho, 90, Centro, CEP 20211-340, Rio de Janeiro, RJ.

E-mail: rom_br@yahoo.com 